\title{
Efficacy of Oral Brush Biopsy in Potentially Malignant Disorder Management
}

\author{
$M$ L Goodson ${ }^{1,2}$ \\ D R Smith ${ }^{2}$ \\ P J Thomson ${ }^{1^{*}}$
}

${ }^{1}$ Oral \& Maxillofacial Surgery, School of Dentistry, University of Queensland, Oral Health Centre, Queensland 4006, Australia.

${ }^{2}$ Newcastle University Medicine Malaysia, 79200 Iskandar Puteri, Johor, Malaysia.

*Corresponding Author - peter.thomson@uq.edu.au

Fax: +61733658118

Keywords: Potentially Malignant Disorders, Diagnosis, Outcome 


\begin{abstract}
Background: Oral potentially malignant disorders harbour unpredictable risk for squamous cell carcinoma development. Current management requires tissue biopsy for histopathology characterisation, dysplasia grading and targeted intervention to 'high risk' lesions, although evidence-based guidelines are limited and diagnoses subjective. This study investigated the use of adjunctive oral brush biopsy techniques during the management of potentially malignant disorders in a UK hospital population.
\end{abstract}

Methods: Retrospective review of a 310 PMD patient cohort presenting to Maxillofacial Surgery in Newcastle upon Tyne with new, single-site lesions between December 2009 and May 2014. Patients underwent Orcellex® brush biopsy and liquid-based cytology examination in addition to conventional biopsy techniques, with management proceeding along established care pathways. Patient demographics, cytology data, most significant histopathology diagnoses and clinical outcome were all documented at the study census date (31.12.15).

Results: 170 male \& 140 female patients (age range 18-91yrs), exhibiting primarily leukoplakia (86.5\%) at floor of mouth and ventro-lateral tongue sites (44.9\%), were identified. Management comprised: observation (49.7\%), laser surgery (44.9\%), antifungal treatment (3.5\%), and Head \& Neck clinic referral following cancer diagnosis (1.9\%). Clinical outcomes were: disease free (51.3\%), persistent PMD (42.3\%) and malignant transformation (6.4\%). Histology and cytology diagnoses strongly correlated $(r=0.305)$. Treatment modality, lesion site, histology and cytology diagnoses were the best predictors of clinical outcome.

Conclusions: Orcellex® brush cytology provides reliable diagnoses consistent with conventional histopathology and offers less invasive, adjunctive assessment appropriate for long-term monitoring of patients in specialist clinics. 


\section{Introduction}

Oral squamous cell carcinoma (SCC) may be preceded by potentially malignant disorders (PMD), distinct mucosal lesions such as leukoplakia, erythroplakia, or erythroleukoplakia, or more widespread disorders such as proliferative verrucous leukoplakia $(P V L)^{1,2}$. Characteristic of $P M D$ is the variable histopathological presence of epithelial disorganisation and dysmaturation which is characterised microscopically as dysplasia ${ }^{1,2}$. Whilst the natural history of PMD remains poorly understood, recent systematic reviews have estimated a $12 \%$ cancer risk over a mean transformation time of 4.3 years $^{3}$. Early diagnosis and intervention during the progression of worsening dysplasia towards SCC thus has potential to improve prognosis and reduce morbidity ${ }^{1}$.

Despite the ability to identify PMD in patients, assessment techniques are highly subjective and clinicians are unable to predict lesion behaviour or quantify individual risk for malignant transformation ${ }^{1}$. Contemporary PMD management is based upon incision biopsy for histological confirmation followed by lesion excision, often via $\mathrm{CO}_{2}$ laser, for definitive diagnosis and treatment of 'high risk' lesions ${ }^{4,5,6}$. Tissue biopsy and histopathological grading of dysplasia, although remaining the gold standard for PMD diagnosis, has been criticised as invasive, expensive, subjective and ultimately unrepresentative, especially for large, multi-focal lesions ${ }^{1,2}$.

Non-invasive adjunctive diagnostic tools were proposed to improve detection of early signs of cancer change in oral mucosa. These include vital staining, optical imaging and brush biopsy cytology although reports of effectiveness are anecdotal, there is criticism they rarely reveal features not demonstrable by conventional techniques and their role in contemporaneous clinical practice remains unclear ${ }^{7,8}$.

Cytodiagnosis is the technique whereby individual cells are loosened from their tissue of origin and transferred to a cytology slide for microscopic examination. Whilst effective sampling of exfoliated oral epithelial cells for analysis has proved unreliable, newer collection devices including a range of 'cytobrushes' have been developed to facilitate full-thickness sampling of stratified epithelium ${ }^{9}$. The Orcellex $®$ brush (Rovers Medical Devices BV., the Netherlands) has a specially designed head comprising 5 segments of high-density fibres designed for optimal cell collection, 
storage and predictable release of cells from multiple layers of oral epithelium (Figure 1A). In clinical practice, the brush head is firmly placed against the mucosa and rotated 10 times (Figure 1B), then detached and transferred in BD SurePath ${ }^{\mathrm{TM}}$ ethanol-based preservative fluid for laboratory transfer. The use of liquid-based cytology (LBC), rather than conventional glass slide smears, improves cell distribution and produces thin layer preparations (Figure 1C). Whilst preliminary trials on the use of the Orcellex® brush have been reported ${ }^{9-11}$, the accuracy of brush cytology requires formal assessment as an objective tool during long term PMD management.

The aim of this study was thus to review the efficacy of adjunctive oral brush biopsy techniques during the diagnosis and interventional management of PMD and to assess the accuracy and potential predictive value of the technique in direct comparison to conventional clinico-pathological data.

\section{Materials and Methods}

\section{Patients and treatment}

Caldicott Approval from Newcastle University / Newcastle upon Tyne Hospitals NHS Foundation Trust facilitated anonymized, retrospective data collection from medical records, operating logs, cytology and pathology reports from PMD patients treated by the senior author (PJT) and attending Newcastle Dental and Royal Victoria Infirmary hospitals between December 2009 and May 2014. Inclusion criteria required new, untreated single-site PMD where Orcellex ${ }^{\circledR}$ brush biopsy and liquidbased cytology (LBC) were performed in addition to conventional biopsy procedures. Patient management proceeded along established PMD care pathways ${ }^{4}$. Demographic and clinico-pathological data obtained for each patient comprised: age, sex, clinical appearance and site of oral lesion, cytology and histopathology diagnoses from original reports, and clinical outcome documented on the study census date (31 December 2015). Patients with previous or multi-focal PMD, and those with a history of OSCC or head and neck radiotherapy were excluded.

\section{Cytology}


Patients underwent Orcellex ${ }^{\circledR}$ brush biopsy as illustrated in Figure 1. Cytology specimens were processed and stained using the BD Prep Stain ${ }^{\mathrm{TM}}$ automated process in which, following density gradient centrifugation to enrich the cellular sample and remove non-diagnostic debris, discretely stained, thin-layer slide preparations were produced. Each specimen was stained with Papanicolau and Periodic Acid Schiff. Specimen reporting was carried out by experienced cytologists applying the Bethesda guidelines for liquid-based squamous cellularity ${ }^{12}$; discussion and consensus grading was carried out when required. Cells were described as normal (including normal and hyperkeratosis categories), exhibiting mild, moderate or severe dyskaryosis, or suggestive of SCC; inflammatory cells and candida were noted as appropriate.

\section{Histopathology}

Incision biopsies were performed under the direction of PJT and excision specimens obtained following interventional laser treatment. Laser surgery was performed by PJT, or by colleagues working under direct supervision, according to previously detailed protocols within 6-12 weeks following lesion presentation to avoid disease progression $^{4-6}$. Formalin-fixed biopsy specimens underwent standardized histopathology examination by experienced oral pathologists working to agreed diagnostic criteria and using the World Health Organisation (WHO) classification to grade tissue as normal (including hyperkeratosis), mild, moderate and severe dysplasia, carcinoma-in-situ (CiS) or SCC. The most severe histopathological diagnosis obtained from original incision or excision biopsy reports was recorded for each patient. In addition, the presence of lichenoid inflammation (LI), and diagnoses of PVL and candida infection were recorded.

\section{Statistical Analysis}

Descriptive statistics were used to detail patient demography, clinical features, cytological and histopathological diagnoses, treatment interventions and follow-up data. Clinical outcomes were stratified into disease-free or further disease (persistent or SCC development) categories and both univariate and multivariate logistic regression analyses were performed. Brush cytology and most significant histopathology diagnoses were treated as categorical variables, and Cramer's V assessment of correlation undertaken. Sensitivity scores, whereby cytology and 
histopathology diagnoses were coded as binary variables, were calculated. In relation to SCC, calculations of sensitivity (proportion of correctly identified positive results), specificity (proportion of correctly identified negative results), and positive and negative predictive values for the Orcellex ${ }^{\circledR}$ brush were determined. Statistical analyses were performed using SPSS, version 19.0 (Statistical Package for the Social Sciences, Chicago, IL, USA).

\section{Results}

Table 1 summarises study results: 310 patients met the search criteria, 140 men (mean age 57.8 years) and 170 women (mean age 59.8 years). Of the 310 lesions assessed, 268 presented as leukoplakias (86.5\%), with erythroleukoplakia (27) and erythroplakia (15) less common. Floor of mouth and ventro-lateral tongue together comprised the most commonly affected site in 139 cases (44.9\%).

All brush cytology samples were of diagnostic quality with 164 specimens reported as 'normal', 137 displaying variable severity of dyskaryosis and 9 suggestive of SCC. Histopathological diagnoses, based upon the most significant abnormality observed, reported 165 'normal' specimens with 131 exhibiting dysplasia and 14 CiS or invasive SCC. Review of diagnostic categories listed in Table 1 and the cytology versus histopathology cross-tabulation presented in Table 2 reveal a very similar distribution; correlation coefficient $r=0.305$ (Cramer's $V$ association) confirmed a moderately strong relationship between results. Whilst reasonable sensitivity and positive predictive values for brush cytology to detect SCC were seen, specificity and negative predictive values were much higher; Table 3.

Candida hyphae were identifiable in both cytology and histology specimens (10\% and $4.8 \%$ respectively), but features of $\mathrm{LI}(28.7 \%)$ and PVL (11.3\%) were only characterised by histopathological examination.

154 patients (49.7\%) were managed by clinical observation alone (principally 'normal' or 'mild dyskaryosis/dysplasia' cases), whilst 139 (44.9\%) underwent laser surgery (usually excision biopsy); medical (anti-fungal) treatment or referral to Head \& Neck oncology clinics was the principal treatment modality in 17 cases (5.4\%).

Patient follow-up ranged from 17 to 72 months (mean 57.9 months). Clinical outcome data showed 159 patients (51.3\%) to be free of clinically observable 
mucosal disease at study census date, whilst 151 (48.7\%) exhibited persistent disease or had undergone SCC transformation. Review of clinico-pathological variables potentially influencing clinical outcome (listed in Table 4 and presented graphically in Figure 2) suggested that treatment modality (in particular the absence of laser treatment), lesion site, histopathology and cytology diagnoses were the best predictors of developing further disease; histopathology exerted an $11 \%$ influence, whilst cytology $7 \%$ suggesting histopathology to be a 1.6 times better predictor than cytology. In contrast, patient age, sex, lesion appearance, and the presence of candida, LI or PVL were all less predictive of outcome.

\section{Discussion}

This paper analysed a cohort of 310 treated PMD patients examined by Orcellex ${ }^{\circledR}$ brush biopsy in addition to conventional histopathology, and confirmed diagnostic reliability together with a potential predictive role. The majority of PMD lesions were leukoplakias on floor of mouth and ventro-lateral tongue sites, with nearly half exhibiting dysplasia or SCC and undergoing interventional laser treatment, consistent with our previously reported patient cohorts ${ }^{4-6}$. In contrast, recent cytology studies have involved less specific mucosal disease, smaller patient numbers and no clinical outcome data ${ }^{13-16}$. Whilst we previously compared cytology with incision biopsy diagnoses for 224 patients in an Orcellex® brush trial, many of those lesions were not dysplastic, no excision biopsies were studied and no treatment or long term follow-up data were available ${ }^{11}$.

The Orcellex $^{\circledR}$ brush appears a useful adjunctive diagnostic technique: readily available in clinic, easy to use, minimally invasive and efficient in cell collection ${ }^{11,13}$. From a clinician perspective, the brush has an optimal design facilitating application to oral sites. Patients report a preference for brush in contrast to conventional techniques requiring local anaesthetic, scalpel or punch instrumentation and suturing $^{11}$. The brush provides trans-epithelial sampling of basal, para-basal and superficial cell layers important for cytology grading, especially relevant in thickened keratin layers characteristic of leukoplakia, the commonest PMD.

A further advantage of LBC is the option to perform additional analyses such as immunocytochemistry, HPV testing, DNA-ploidy, or the measurement of biomarkers in cells left over in collection fluid after diagnostic smear preparation ${ }^{9,17}$; we have 
previously demonstrated high Ki-67 labelling in PMD lesions with aggressive disease, so there may be an enhanced prognostic role for future labelling studies (Figure 1D) $)^{18}$.

Whilst significant correlation was seen between cytology and histopathology diagnoses (134 study samples matched precisely; Table 2), direct comparison may not always be feasible due to variations in cytology terminology and lack of available tissue structure, which is essential for histopathological characterisation of $\mathrm{LI}$ and $\mathrm{PVL}$ and pertinent for patients developing further or persistent $\mathrm{PMD}^{6}$.

Prediction of clinical outcome remains elusive in clinical practice, especially for individual patient management ${ }^{4-6}$. In this study the choice of treatment modality, in particular not administering laser surgery, was seen on multivariate analysis to be a predictor of further disease; probably unsurprising due to laser's efficacy in PMD excision $^{6}$. Whilst histopathology diagnoses were better predictors of outcome than cytology, brush biopsy appeared more accurate than traditional clinico-pathological data such as patient age, sex, clinical lesion appearance or additional features such as candidal infection, $\mathrm{LI}$ and PVL.

Malignant transformation rates for PMD vary worldwide, with quoted ranges varying between $0.1 \%$ and $40 \%$ and an overall mean of $12 \%$, although we have highlighted that up to $12 \%$ of PMDs may already harbour invasive SCC on initial presentation ${ }^{6}$. LBC, in the absence of histological confirmation, can only raise suspicion that abnormal cells have arisen in an invasive SCC. It is notable, however, that we found the number of cytology and histopathology SCC diagnoses similar at 9 and 14 cases respectively (the latter included 4 CiS cases; Table 1).

Whilst the sensitivity and positive predictive values for brush detected SCC were reasonable (60\% and $67 \%$ respectively), specificity and negative predictive values showed high credibility (99\%). Orcellex ${ }^{\circledR}$ brush biopsy therefore, whilst not a substitute for conventional biopsy and histological examination, offers additional 'screening' opportunities for abnormal cell identification especially for sampling of large, widespread or multi-focal lesions during long-term monitoring and active surveillance of patients post-PMD treatment ${ }^{6}$. 
This clinico-pathological review took place within a single-centre, specialist PMD service and was not a prospective, randomized controlled trial. Nonetheless, the study supports Orcellex® brush biopsy and LBC as practical, effective and reliable adjunctive diagnostic techniques during management of a 310 PMD patient cohort followed for up to 6 years in a hospital setting.

\section{Acknowledgements}

The authors acknowledge the invaluable assistance of colleagues in the Departments of Cellular Pathology, in particular Dr Viney Wadehra and Dr Sarah Johnston for their expertise in diagnostic cytopathology, Medical Physics, Anaesthesia and Peri-Operative Care at the Newcastle upon Tyne Hospitals NHS Foundation Trust, without whom this clinical work and study would not have been possible.

\section{Funding}

None declared.

\section{Competing Interests}

None declared.

\section{Ethical Approval}

Newcastle University / Newcastle upon Tyne Hopsitals NHS Foundation Trust Caldicott Guardian Approval for Anonymised Patient Data Collection \& Retrospective Review of Hospital records ID 4143 (2015) 


\section{References}

1. Thomson PJ. Oral Carcinogenesis. In: PJ Thomson (ed) Oral Precancer Diagnosis and Management of Potentially Malignant Disorders. Chichester: WileyBlackwell; 2012. p31-47.

2. van der Waal I. Potentially malignant disorders of the oral and oropharyngeal mucosa; terminology, classification and present concepts of management. Oral Oncology 200945 : 317-323.

3. Mehanna HM, Rattay T, Smith J, McConkey CC. Treatment and follow-up of oral dysplasia - a systematic review and meta-analysis. Head \& Neck 200931 : 16001609.

4. Thomson PJ, Wylie J. Interventional laser surgery: an effective surgical and diagnostic tool in oral precancer management. International Journal of Oral \& Maxillofacial Surgery $200231: 145-153$.

5. Diajil AR, Robinson CM, Sloan P, Thomson PJ. Clinical outcome following oral potentially malignant disorder treatment: a 100 patient cohort study. International Journal of Dentistry 2013 Article ID 809248, 8 pages http://dx.doi.org/10.1155/2013/809248.

6. Thomson PJ, Goodson ML, Cocks K, Turner JE. Interventional laser surgery for oral potentially malignant disorders: a longitudinal patient cohort study. International Journal of Oral \& Maxillofacial Surgery 201746 : 337-342.

7. Messadi DV. Diagnostic aids for detection of oral precancerous conditions. International Journal of Oral Science 20135 : 59-65.

8. Thomson PJ, Sloan P, Wadehra V, Robinson CM, Johnson SJ, Mowatt A, Goodson ML. Diagnostic adjunctive techniques and the management of oral potentially malignant disorders. Oral Surgery, Oral Medicine, Oral Pathology, Oral Radiology 2015 119: e212 
9. Kujan O, Desai M, Sargent A, Bailey A, Turner A, Sloan P. Potential applications of oral brush cytology with liquid-based technology: results from a cohort of normal oral mucosa. Oral Oncology 200642 : 810-818.

10. Goodson ML, Wadhera V, Johnson S, Sloan P, Robinson CM, Thompson WR, Thomson PJ. Brush versus scalpel: consensus agreement on Orcellex® brush cytology versus incisional biopsy. Oral Oncology 2013 49: S96-S97 doi:10.1016/j.oraloncology.2013.03.254.

11. Goodson ML, Sloan P, Wadehra V, Johnson SJ, Robinson CM, Aubourg G, Thomson PJ. Orcellex® brush biopsy and liquid-based cytology - assessment of a new diagnostic technique in oral potentially malignant disorder management. Journal of Oral and Maxillofacial Surgery 2014 Photon 117: 224-231 ISJN37924853D666928032014

12. Solomon D. The 2001 Bethesda System: terminology for reporting results of cervical cytology. Journal of the American Medical Association 2002287 : 21142119.

13. Mehrotra R. The role of cytology in oral lesions: a review of recent improvements. Diagnostic Cytopathology 2011 40: 73-83.

14. Mehrotra R, Mishra S, Singh M, Singh M. The efficacy of oral brush biopsy with computer-assisted analysis in identifying precancerous and cancerous lesions. Head \& Neck Oncology 2011 3: 39 doi: 10.1186/1758-3284-3-39.

15. Guneri P, Epstein JB, Kaya A, Veral A, Kazandt A, Boyacioglu H. The utility of toluidine blue staining and brush cytology as adjuncts in clinical examination of suspicious oral mucosal lesions. International Journal of Oral \& Maxillofacial Surgery 2011 40: 155-161

16. Reddy SG, Kanala S, Chigurapati A, Kumar SR, Poosarla CS, Reddy BV. The sensitivity and specificity of computerized brush biopsy and scalpel biopsy in diagnosing oral premalignant lesions: A comparative study. Journal of Oral \& Maxillofacial Pathology 2012 16: 349-353.

17. Yang Y, Rhodus NL, Ondrey FG, Wuertz BRK, Chen X, Zhu Y, Griffin TJ. Quantitative proteomic analysis of oral brush biopsies identifies secretory leukocyte protease inhibitor as a promising, mechanism-based oral cancer biomarker. PLoS ONE 9(4): e95389 doi:10.1371/journal.pone.0095389.

18. Thomson PJ, Goodson ML, Booth C, Cragg N, Hamadah O. Predicting recurrence after treatment of oral precancer: Use of cell cycle analysis. British Journal of Oral \& Maxillofacial Surgery 2008 46: 370-375. 
Table 1: Patient Demographic, Clinico-Pathological and Outcome Data for PMD Patients (Number $=310$ ).

\begin{tabular}{|c|c|}
\hline Patient Sex & No. (\%) \\
\hline Male & $170(54.8 \%)$ \\
\hline Female & $140(45.2 \%)$ \\
\hline Patient Age & Mean Years (SD) \\
\hline Male & $57.8(12.38)$ \\
\hline Female & $59.8(12.56)$ \\
\hline Lesion & No. (\%) \\
\hline Leukoplakia & $268(86.5 \%)$ \\
\hline Erythroleukoplakia & $27(8.7 \%)$ \\
\hline Erythroplakia & $15(4.8 \%)$ \\
\hline Site & No. (\%) \\
\hline Floor Of Mouth & $62(20 \%)$ \\
\hline Lateral Tongue & $60(19.4 \%)$ \\
\hline Buccal Mucosa & $55(17.7 \%)$ \\
\hline Palate & $38(12.3 \%)$ \\
\hline Alveolus & $24(7.7 \%)$ \\
\hline Gingiva & $18(5.8 \%)$ \\
\hline Ventral Tongue & $17(5.5 \%)$ \\
\hline Dorsum of Tongue & $13(4.2 \%)$ \\
\hline Labial Mucosa & $11(3.5 \%)$ \\
\hline Fauces / Retromolar Region & $7 \quad(2.3 \%)$ \\
\hline Labial Commissure & $5(1.6 \%)$ \\
\hline Brush Cytology Diagnosis & No. (\%) \\
\hline Normal & $164(52.9 \%)$ \\
\hline Atypia / Mild Dyskaryosis & $79(25.5 \%)$ \\
\hline Moderate Dyskaryosis & $26(8.4 \%)$ \\
\hline Severe Dyskaryosis & $32(10.3 \%)$ \\
\hline ? SCC & $9(2.9 \%)$ \\
\hline
\end{tabular}




\begin{tabular}{|l|r|}
\hline Histopathology Diagnosis (Most Significant) & No. (\%) \\
\hline Normal & $165(53.2 \%)$ \\
\hline Mild Dysplasia & $74(23.9 \%)$ \\
\hline Moderate Dysplasia & $34(11 \%)$ \\
\hline Severe Dysplasia & $23(7.4 \%)$ \\
\hline Carcinoma in Situ / SCC & $14(4.5 \%)$ \\
\hline Lichenoid Inflammation & No. (\%) \\
\hline Yes & $89(28.7 \%)$ \\
\hline No & $221(71.3 \%)$ \\
\hline PVL & No. (\%) \\
\hline Yes & $34(11.3 \%)$ \\
\hline No & $276(91.7 \%)$ \\
\hline Candida (Cytology Diagnosis) & No. (\%) \\
\hline Yes & $31(10 \%)$ \\
\hline No & $279(90 \%)$ \\
\hline Candida (Histopathology Diagnosis) & No. (\%) \\
\hline Yes & $15(4.8 \%)$ \\
\hline No & $295(95.2 \%)$ \\
\hline Treatment Intervention & No. (\%) \\
\hline Observation & $154(49.7 \%)$ \\
\hline Laser Treatment & $139(44.9 \%)$ \\
\hline Medical Treatment & $11(3.5 \%)$ \\
\hline Referral to Head \& Neck MDT & $6(1.9 \%)$ \\
\hline Clinical Outcome & $159(51.3 \%)$ \\
\hline Disease Free & $131(42.3 \%)$ \\
\hline Persistent Disease & $20(6.4 \%)$ \\
\hline Malignant Transformation & \\
\hline & \\
\hline
\end{tabular}

Table 2: Cross-tabulation of the Number of Cases and Sensitivity Scores (parentheses) for Cytology vs. Histopathology Diagnoses.

\begin{tabular}{|ll|l|l|l|l|l|l|}
\hline \multirow{2}{*}{} & \multicolumn{5}{|c|}{ HISTOPATHOLOGY DIAGNOSES } & \multirow{2}{*}{ Total } \\
\cline { 3 - 7 } & & Normal & Mild Dysp & Mod Dysp & Severe Dysp & CiS/SCC & \\
\hline \multirow{2}{*}{ CYTOLOGY } & Normal & $100(0.61)$ & $42(0.57)$ & $13(0.38)$ & $7(0.3)$ & $2(0.14)$ & 164 \\
DIAGNOSES & Mild Dysk & $46(0.28)$ & $17(0.23)$ & $10(0.29)$ & $4(0.17)$ & $2(0.14)$ & 79
\end{tabular}




\begin{tabular}{|l|l|l|l|l|l|l|l|}
\hline Moderate Dysk & $7(0.04)$ & $6(0.08)$ & $5(0.15)$ & $5(0.22)$ & $3(0.21)$ & $\mathbf{2 6}$ \\
Severe Dysk & $11(0.07)$ & $8(0.11)$ & $6(0.18)$ & $6(0.26)$ & $1(0.07)$ & $\mathbf{3 2}$ \\
?SCC & $1(0.01)$ & $1(0.01)$ & $0(0)$ & $1(0.04)$ & $6(0.43)$ & $\mathbf{9}$ \\
& & & & & & & \\
Total & $\mathbf{1 6 5}$ & $\mathbf{7 4}$ & $\mathbf{3 4}$ & $\mathbf{2 3}$ & $\mathbf{1 4}$ & $\mathbf{3 1 0}$ \\
\hline
\end{tabular}

For computation of sensitivity scores, each cytology and histopathology diagnoses were coded as binary variables (not present/present).

Abbreviations: Mod $=$ Moderate $;$ Dysk = Dyskaryosis $;$ Dysp $=$ Dysplasia $;$ CiS = Carcinoma in Situ; SCC $=$ Squamous Cell Carcinoma

Table 3: Sensitivity, Specificity, Positive and Negative Predictive Values for Orcellex® Brush Cytology detecting Squamous Cell Carcinoma (SCC), assuming Histopathology as 'gold standard'.

\begin{tabular}{|l|l|}
\hline Test & $\%$ \\
\hline Sensitivity & 60 \\
\hline Specificity & 99 \\
\hline Positive Predictive Value & 67 \\
\hline Negative Predictive Value & 99 \\
\hline
\end{tabular}

Table 4: Analysis of clinico-pathological factors influencing further disease status.

\begin{tabular}{|l|l|l|}
\hline Clinico-Pathological Factor & $\begin{array}{l}\text { Univariate Analysis } \\
\text { (P value) }\end{array}$ & $\begin{array}{l}\text { Multivariate Model } \\
\text { (P value) }\end{array}$ \\
\hline Age & ----- & Linear: 0.67; Non-Linear 0.79 \\
\hline Sex & 0.82 & 0.52 \\
\hline Site & $<0.001$ & 0.14 \\
\hline Histology & $<0.001$ & 0.17 \\
\hline Cytology & 0.15 & 0.36 \\
\hline Clinical Appearance & 0.75 & 0.98 \\
\hline PVL & 0.23 & 0.78 \\
\hline Lichenoid Inflammation & 0.28 & 0.51 \\
\hline Candida & 0.17 & 0.26 \\
\hline
\end{tabular}


Figure 1: (A) Orcellex® brush head in close-up showing high-density fibres for optimal cell collection (B) brush in use clinically for floor of mouth sampling, (C) Papanicolaou stained thin-layer LBC preparation under microscopy (x40) and (D) high Ki67 labelling seen in dyskaryosis (x100).

Figure 2: Contribution of independent variables in predicting outcome from the multiple logistic regression model. 